\title{
Anion charge and lattice volume maps for searching lithium superionic conductors
}

\author{
Zhenming $\mathrm{Xu}^{\mathrm{a}, \mathrm{b}}$, Hong $\mathrm{Zhu}^{\mathrm{a}}{ }^{*}$ \\ a. University of Michigan-Shanghai Jiao Tong University Joint Institute, Shanghai Jiao Tong \\ University, 800 Dongchuan Road, Shanghai, 200240, China \\ b. Harvard John. A. Paulson School of Engineering and Applied Sciences, Harvard University, \\ 29 Oxford Street, Cambridge, Massachusetts, 02138, USA
}

\begin{abstract}
The effects of anion charge and lattice volume (lithium-anion bond length) on lithium ion migration have been investigated by utilizing the density functional theory calculations combined with the anion sublattice models, e.g. $f c c, h c p$ and $b c c$. It is found that the anion charge and lattice volume have great impacts on the activation energy barrier $\left(E_{a}\right)$ of lithium ion migration, which is validated by some reported sulfides. For the tetrahedrally occupied lithium, the less negative anion charge is, the lower the lithium ion migration barrier is likely to be. While for the octahedrally occupied lithium, the more negative anion charge is, the lower the lithium ion migration barrier is. There are opposite effects of anion charge on $E_{a}$ and optimum lattice volumes for minimum $\mathrm{E}_{\mathrm{a}}$ of lithium ion migration along the Tet-Oct-Tet and Oct-Tet-Oct pathways in the hcp-type sublattices. Based on the full understandings of anion sublattice model, general design strategies for developing lithium superionic conductors were proposed. Adjusting the electronegativity difference between the anion element and non-mobile cation element by selecting the most suitable non-mobile cation element without changing the crystal structure sublattice can achieve low $E_{a}$ for lithium ion migration. For the desired lithium superionic conductors with tetrahedrally occupied lithium ions, the fine non-mobile cation element should give preferences to those elements located at the right top of the periodic table of elements with large electronegativities. For the lithium superionic conductors with octahedrally occupied lithium ions, the fine non-mobile cation element should give preferences to the elements located at the left bottom of the periodic table with small electronegativities.
\end{abstract}

Keywords: ionic conductor; lithium ion migration; anion sublattice; anion charge; lattice volume

${ }^{*}$ Corresponding author:

E-mail: hong.zhu@sjtu.edu.cn 


\section{Introduction}

Today, lithium ion battery (LIB) techniques provide much conveniences for people's life, powering the portable electronic devices ${ }^{1}$. However, we encounter the safety issues when using the commercial LIBs in the application scenarios of the large-scale energy storage in electric vehicles, because of the flammability of liquid organic electrolyte ${ }^{2-3}$. Fortunately, replacing the currently employed flammable organic electrolytes with the inflammable solid-state electrolyte (SSE) materials and matching with the lithium metal anodes to construct the all-solid-state lithium ion batteries (ASSLIBs) not only solve the safety issues, but also drastically promote the energy density of $\mathrm{LIBs}^{4-6}$, making electric cars run farther. Correspondingly, high room temperature lithium ionic conductivities $\left(10^{-3}-10^{-2} \mathrm{~S} \mathrm{~cm}^{-1}\right)$ of SSE materials are the essential prerequisite for constructing ASSLIB systems. At present, some lithium superionic conductors, e.g. $\mathrm{Li}_{7} \mathrm{La}_{3} \mathrm{Zr}_{2} \mathrm{O}_{12}{ }^{7}$, $\mathrm{Li}_{1+\mathrm{x}} \mathrm{Al}_{\mathrm{x}} \mathrm{Ti}_{2-\mathrm{x}}\left(\mathrm{PO}_{4}\right)_{3}{ }^{8}$ oxides and $\mathrm{Li}_{10} \mathrm{GeP}_{2} \mathrm{~S}_{12}$ (LGPS) ${ }^{9}, \mathrm{Li}_{7} \mathrm{P}_{3} \mathrm{~S}_{11}{ }^{10}$ sulfides, have been widely studied and successfully developed as the SSE materials, and the state-of-the-art room temperature ionic conductivities of $12-17 \mathrm{mS} \mathrm{cm}{ }^{-1}$ are experimentally realized in $\mathrm{Li}_{10} \mathrm{GeP}_{2} \mathrm{~S}_{12}$ and $\mathrm{Li}_{7} \mathrm{P}_{3} \mathrm{~S}_{11}$ sulfides. To efficiently exploit more advanced lithium superionic conductors, it's quite necessary to deeply understand the fast ion migration mechanism in the state-of-the-art superionic conductors, and even propose proper design strategies.

Among the various studies on understanding fast ion migration, the topology analysis of ion migration pathways is possibly the most common method ${ }^{11-13}$. Eremin et al. applied the geometrical-topological approach based on the Voronoi partition theory to perform the high-throughput search for new potential potassium solid electrolytes ${ }^{14-15}$. Ceder et al. calculated the topology features of the anion sublattice structures of various lithium sulfides, revealed a fundamental relationship between anion packing pattern and lithium ionic transport, and eventually proposed the design concept of body-centered cubic-like anion sublattice with face-shared tetrahedral lithium sites for fast ion migration ${ }^{11}$. Subsequently, they utilized this design concept and computationally predicted a new lithium superionic conductor, $\mathrm{Li}_{2} \mathrm{Zn}_{0.5} \mathrm{PS}_{4}$, with exceptionally high lithium ion conductivity at room temperature ${ }^{16}$. In addition, Mo et al. pointed out the unique feature of the common lithium superionic conductors with abundant enlarged lithium sites caused by large local spaces (fractionally occupied lithium sites) in the crystal structural sublattice, and identified many new structures as fast lithium-ion conductors by the high-throughput screening combined with this quantified feature ${ }^{12}$. However, these understandings based on the topology of lithium migration channels are insufficient to explain the enhanced ionic conductivity by $\mathrm{Cl}$ doping and 
isovalent cation substitution in LGPS families and lithium-argyrodites. The Cl-doped silicon-based $\mathrm{Li}_{9.54} \mathrm{Si}_{1.74} \mathrm{P}_{1.44} \mathrm{~S}_{11.7} \mathrm{Cl}_{0.3}$ shows a quite high ionic conductivity of $25 \mathrm{mS} / \mathrm{cm}$ at room temperature (twice that of the original LGPS) ${ }^{17}$, in which thoroughly substituting Ge in LGPS with Si and partially replacing $\mathrm{S}$ with $\mathrm{Cl}$ have negligible influence on the lattice constants $(a=8.71 \AA$ and $c=$ $12.57 \AA$ of $\mathrm{Li}_{9.54} \mathrm{Si}_{1.74} \mathrm{P}_{1.44} \mathrm{~S}_{11.7} \mathrm{Cl}_{0.3}{ }^{17} v s . a=8.717 \AA$ and $c=12.63 \AA$ of LGPS ${ }^{9}$ ), the local atom arrangements and even the morphologies of lithium ion migration channel. Thus, there would be other important factors influencing the lithium ionic conductivity in addition to the topology-based structure features.

In ionic materials, the total energy landscape of $\mathrm{Li}$ ion in an ionic solid can be described by the classical Coulomb-Buckingham potential model ${ }^{18-19}$, which can be further divided into the short-ranged Li-anion Pauli repulsive interaction, short-ranged Li-anion electrostatic attractive interaction, and long-ranged Li-cation electrostatic repulsive interaction. The resulting total energy landscapes are mainly set by the short-ranged Li-anion interactions, so Li ion migration in an ionic compound can be approximately reduced to Li ion migration in an anion sublattice model ${ }^{13}$. It is also noted that the Li-cation repulsive interactions also contribute to the total energy landscape to some extents, and the weight of the Li-cation interaction in setting the total energy landscape is set by the arrangements and valance states of cation. As mentioned in some previous work, the monovalent halogen anions have weaker interactions with lithium ions than divalent sulfur or oxygen anions ${ }^{20-21}$. It's worth noting that W.G Zeier et al. have demonstrated the influence of lattice dynamics and the softness of the lattice on ionic transport ${ }^{22-25}$. Lattice dynamic is the manifestation of bonding interaction in materials, not the fundamental factors of determining bonding interaction. Yet, as far as we know, there is no systematical understanding of the Li-anion interaction dependent lithium ion occupation and lithium ion migration in compounds with different type anion sublattices, which remains to be uncovered as regards this issue. Therefore, this study aims to fill the existing knowledge gaps regarding the effect of Li-anion interaction on lithium ion migration in solids.

In the most crystalline lithium compounds, lithium ions occupy the tetrahedral (Tet) or octahedral $(O c t)$ sites, forming the stable tetrahedral or octahedral lithium-anion polyhedrons, and the anion packing modes of one-half lithium compounds could be approximately classified into the face-centered cubic $(f c c)$, body-centered cubic $(b c c)$ and hexagonal close-packed $(h c p)$ sublattices ${ }^{11}$. The anion sublattices of $\mathrm{LiCoO}_{2}, \mathrm{Li}_{2} \mathrm{MnO}_{3}, \mathrm{Li}_{4} \mathrm{Ti}_{5} \mathrm{O}_{12}, \mathrm{Li}_{2} \mathrm{~S}, \mathrm{LiTiS}_{2}$ and $\mathrm{Li}_{3} \mathrm{YBr}_{6}$ can be exactly matched to the $f c c$ types. In $\mathrm{Li}_{7} \mathrm{P}_{3} \mathrm{~S}_{11}$ and $\mathrm{Li}_{10} \mathrm{GeP}_{2} \mathrm{~S}_{12}$, the sulfur anion sublattices can be roughly mapped to the $b c c$ lattices with some distortions. In $\gamma-\mathrm{Li}_{3} \mathrm{PS}_{4}$ and $\mathrm{Li}_{4} \mathrm{GeS}_{4}$, the sulfur anion 
sublattices can be closely matched to the $h c p$ arrays ${ }^{11}$. Our previous studies of the $f c c$ anion sublattices show that the anion charge and lattice volume have great impacts on the stability of lithium ion occupation, and lithium ion migration ${ }^{26-27}$. Considering the excellent lithium ion migration usually occurring in the sulfide-type superionic conductors with the $b c c$ and $h c p$ sulfur anion sublattices, such as $\mathrm{Li}_{7} \mathrm{P}_{3} \mathrm{~S}_{11}$ and $\mathrm{Li}_{10} \mathrm{GeP}_{2} \mathrm{~S}_{12}$, in this work, we shed lights on the effects of the anion charge and lattice volume dependent Li-anion interaction on the lithium ion occupation and lithium ion migration along different pathways in the $b c c$ and $h c p$ anion sublattices. By the depicted anion charge and lattice volume maps, we suggested some design strategies for different diffusion path to lower activation energy barrier for lithium ion migration in terms of anion charge control and element selection.

\section{Computational methodologies}

All calculations were performed by using the Vienna $a b$-initio Simulation Package (VASP) software based on the projector augmented wave (PAW) method ${ }^{28}$ in the sublattice of the density functional theory $(\mathrm{DFT})^{29}$. The Perdew-Burke-Ernzerhof (PBE) exchange functional ${ }^{30}$ in the sublattice of generalized gradient approximation $(\mathrm{GGA})^{31}$ was utilized to solve the Schrödinger's equation of the quantum states of electrons. The energy cutoff of plane-wave is $500 \mathrm{eV}$. The convergence criteria of energy and force are $10^{-5} \mathrm{eV} /$ atom and $0.01 \mathrm{eV} / \AA$, respectively. The Monkhorst-Pack method ${ }^{32}$ with $4 \times 4 \times 4$ and $4 \times 4 \times 2 \quad k$-point mesh were employed for the Brillouin zone sampling of the body-centered cubic and hexagonal close-packed anion sublattices, respectively. The energy landscapes and activation energy barrier of lithium ion migration in the anion sublattices are calculated by the nudged elastic band (NEB) $\operatorname{method}^{33}$. During the NEB calculations, only one lithium ion is allowed to move, while the sulfur(oxygen) anions are fixed in their initial positions, and this approach has been also utilized by Ceder et $\mathrm{al}^{11}$. The anion charges in the anion sublattice are changed by adjusting the uniform background charges of the whole sublattice system.

\section{Results and discussion}

\subsection{Anion charge and lattice volume dependent lithium occupation and migration}


First, to get the reasonable ranges of anion charge and lattice volume for the following NEB calculations, the scatter distributions of the anion Bader charges and lattice volumes of lithium oxides and sulfides were investigated and shown in Figure S1, which were directly obtained from the AFLOW database without any DFT calculation ${ }^{34}$. It can be found that the oxygen anion charges are mainly spread in the range of $[-0.8 e,-1.7 e]$, while the sulfur anion charges are principally distributed in the range of $[-0.7 e,-1.5 e]$. Thereby, a wider anion charge range of $[-0.5 e,-1.8 e]$, simultaneously covering the dominating oxygen and sulfur anion charges, was considered for the following NEB calculations. The lattice volumes of the oxides and sulfides are mainly spread in the ranges of [15 $\AA^{3} /$ atom, $30 \AA^{3} /$ atom] and [30 $\AA^{3} /$ atom, $50 \AA^{3} /$ atom], respectively. In addition, the Bader charges of lithium cation in the lithium oxides and sulfides are dominantly located in the range of $[+0.84 e \sim+0.92 e]$ (Figure S2), which are far narrower than those of anions. It indicates that the charges of lithium ion in different compounds don't change a lot, and thereby can be regarded as a constant.

Then, two artificial $b c c$ - and $h c p$-type anion sublattices with a single lithium cation and 16 anions were built to investigate lithium ion migration in them, as illustrated in Figure 1. Only two chalcogen anion elements (oxygen and sulfur) were considered for the anion sublattice models in view of the fact that the most reported lithium superionic conductors are lithium oxides and sulfides. These sulfur(oxygen) anion sublattice models can allow us directly capture the effects of the Li-anion interaction between lithium cation and its adjacent anion, which fundamentally depend on the anion charge and lithium-anion bond length. Our anion sublattice models are much beyond the bond-valance model for energy barrier calculations with compromised accuracy, due to its strong dependency of the empirical parameters ${ }^{35-36}$. In this work, for the convenience of the NEB energy landscape calculations, we employed the descriptor of anion sublattice volume (averaged to per anion atom, unit cell volume/number of anion) instead of the lithium-anion bond length, because there is a positive relationship between the lattice volume and lithium-anion bond length in the anion sublattice model. There is only one kind of tetrahedral interstitial site in the bcc-type anion sublattice (Figure 1a), corresponding to a Tet-Tet lithium ion migration pathway, and this direct lithium ion hopping between two adjacent Tet sites has been reported to possess the lowest activation barrier and the highest lithium ionic conductivity ${ }^{11}$. For the $h c p$-type anion sublattice model, there are two different kinds of interstitial sites, including the tetrahedral and octahedral interstitial sites (Figure 1b). Thus, the occupied lithium ion in the hcp anion sublattice could migrate 
along three different direct pathways, including Tet-Tet, Oct-Oct and Oct-Tet pathways, and the latter is the half of the Tet-Oct-Tet and Oct-Tet-Oct pathways.

(a)

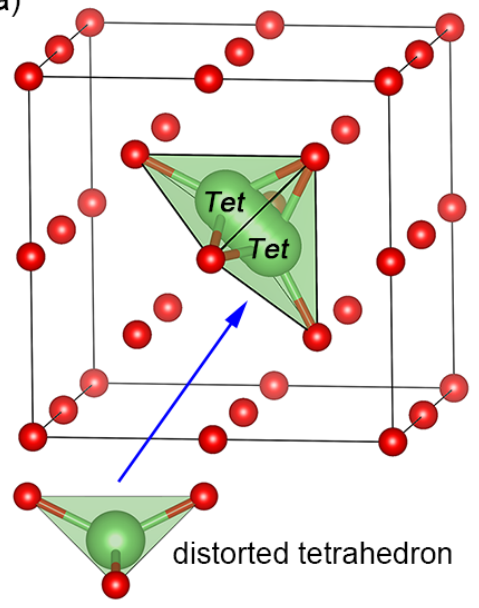

(b)

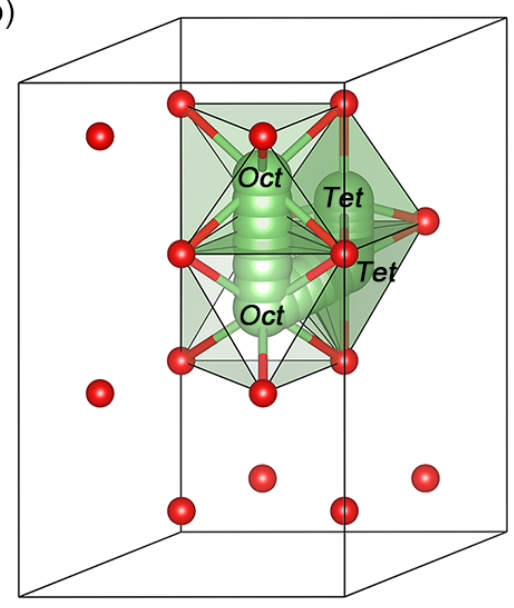

Figure 1. Structural models of (a) lithium ion migration along the Tet-Tet pathway (two adjacent tetrahedral sites) in the bcc-type anion sublattice with a lithium cation and 16 anions, the lithium-anion tetrahedrons in the $b c c$-type anion sublattice are distorted with a csm (continuous symmetry measure) value of 2.29 (a csm value of 0 corresponds to a perfect tetrahedron); (b) lithium ion migration along the Tet-Tet pathway, Oct-Oct pathway (octahedral site to octahedral site), and Oct-Tet pathway in the hcp-type anion sublattice with a lithium cation and 16 anions. The anions are colored red, and lithium ions are colored green, respectively.

\subsection{1 bcc-type anion sublattices}

Then, we performed NEB calculations to get the energy landscapes of lithium ion migration in the $b c c$-type sulfur(oxygen) anion sublattices with respect to anion charge and lattice volume. Lithium ion migration between two face-shared distorted lithium-anion tetrahedral sites (namely Tet-Tet pathway) in the bcc-type anion sublattice passes through a distorted triangular anion bottleneck (Figure 1a), whose relative energy with respect to the Tet site corresponds to the activation energy barrier $\left(E_{a}\right)$ of lithium ion migration. Note, achieving high ionic diffusivity not only needs small $E_{a}$, but also claims large prefactor. Considering the most contribution of small $\mathrm{E}_{\mathrm{a}}$ to high ionic diffusivity and the complex composition of prefactor, in this work we mainly focused on the anion charge and lattice volume dependent $E_{a}$ for lithium ion diffusion. The $E_{a}$ of lithium ion migration in the $b c c$-type sulfur(oxygen) anion sublattices are shown in Figure 2 and S3, respectively. To clearly 
show the $E_{a}$ variations with respect to different anion charges at any constant lattice volume, the heatmaps of Figure 2 and S3 are transformed into the facetgrid plots, as illustrated in Figure S5 and S6, respectively. It can be clearly seen that the anion charge and lattice volume show the similar influences on $E_{a}$ for both sulfur and oxygen anion sublattices. The $E_{a}$ variation ranging from 0 to $0.42 \mathrm{eV}$ in the whole anion charge and lattice volume space of the $b c c$-type oxygen anion sublattice (Figure S3) is much narrower than that of the $f c c$-type oxygen anion sublattice from 0.25 to $1.5 \mathrm{eV}$ (Figure S4a). In addition, the maximum variations of $E_{a}$ with respect to different anion charges at any constant lattice volume are $\sim 0.12$ and $0.07 \mathrm{eV}$, respectively for the oxygen and sulfur anion sublattice (Figure S5 and S6). They indicate that $\mathrm{E}_{\mathrm{a}}$ of lithium ion migration in the bcc-type anion sublattices are not only smaller but also less sensitive to the variations of anion charge than that in the $f c c$-type anion sublattice. It's worth noting that the lithium compounds possessing the $b c c$-type anion sublattices are much fewer than those with the $f c c$ - and $h c p$-anion sublattices (Table S1), although they usually show higher lithium ion conductivities ${ }^{11}$. Overall, the anion charge and lattice volume (lithium-anion bond length) dependent Li-anion interactions have smaller impacts on $\mathrm{E}_{\mathrm{a}}$ of lithium ion migration along the Tet-Tet pathways in the bcc-type anion sublattices. From the energy maps the most important conclusion which can be made is that the activation energy depends more strongly on the lattice volume than on the anion charge.

In the whole anion charge and lattice volume space, the more negative anion charges can lead to slightly higher $\mathrm{E}_{\mathrm{a}}$ for lithium ion migration in the bcc-type anion sublattice along the Tet-Tet pathways at any constant lattice volume (Figure S5 and S6), and the large lattice volumes can reduce $\mathrm{E}_{\mathrm{a}}$ at any constant anion charge (Figure 2 and S3). In short, the little negative anion charges and large lattice volumes can lead to lower $\mathrm{E}_{\mathrm{a}}$. Therefore, the little negative anion charges and large lattice volumes (lithium-anion bond lengths) are essential for achieving fast lithium ion migration in lithium compounds with the $b c c$-type anion sublattices. It's worth noting that these variation trends of $E_{a}$ with respect to anion charge and lattice volume are much more interesting than the absolute values of $E_{a}$. These observed trends are also found in the previous experimental results for some sulfide-type lithium conductors with the $b c c$-type anion sublattices. The higher element electronegativity of $\mathrm{Ge}^{4+}$ vs. $\mathrm{Sn}^{4+}(2.116 \text { vs. 1.877) })^{37}$ lead to less negative sulfur anion charges in $\mathrm{Li}_{10} \mathrm{GeP}_{2} \mathrm{~S}_{12}$ (-0.151e vs. -0.168 e in $\mathrm{Li}_{10} \mathrm{SnP}_{2} \mathrm{~S}_{12}$, Hirshfeld charge $)^{27}$, and the Li-S average bond length of $\mathrm{Li}_{10} \mathrm{GeP}_{2} \mathrm{~S}_{12}$ is $2.585 \AA$, slightly larger than $2.578 \AA$ of $\mathrm{Li}_{10} \mathrm{SnP}_{2} \mathrm{~S}_{12}{ }^{27}$. The relatively less negative sulfur anion charge together with the slightly larger bottleneck area eventually lead to relatively lower $\mathrm{E}_{\mathrm{a}}$ of $\mathrm{Li}_{10} \mathrm{GeP}_{2} \mathrm{~S}_{12}$ compared to $\mathrm{Li}_{10} \mathrm{SnP}_{2} \mathrm{~S}_{12}{ }^{38-39}$. The enhanced ionic conductivities can also be obtained by the monovalent $\mathrm{Cl}$ element doping in lithium sulfides, the $\mathrm{Cl}$-doped 
$\mathrm{Li}_{9.54} \mathrm{Si}_{1.74} \mathrm{P}_{1.44} \mathrm{~S}_{11.7} \mathrm{Cl}_{0.3}$ shows a quite higher ionic conductivity of $25 \mathrm{mS} / \mathrm{cm}$ at room temperature than LGPS ${ }^{17}$, in which thoroughly substituting Ge of LGPS with Si and partially replacing S with $\mathrm{Cl}$ has negligible influence on the lattice constants and even the morphologies of lithium ion migration channel. These consistent variation tendencies of $\mathrm{E}_{\mathrm{a}}$ in the cation-substituted $\mathrm{Li}_{10} \mathrm{MP}_{2} \mathrm{~S}_{12}$ (where $\mathrm{M}=\mathrm{Ge}$ and $\mathrm{Sn}$ ) and chlorine-doped $\mathrm{Li}_{9.54} \mathrm{Si}_{1.74} \mathrm{P}_{1.44} \mathrm{~S}_{11.7} \mathrm{Cl}_{0.3}$ materials are fully consistent with our findings, validating the reasonability of the $b c c$-type anion sublattice model and the corresponding analyses of Li-anion interactions.

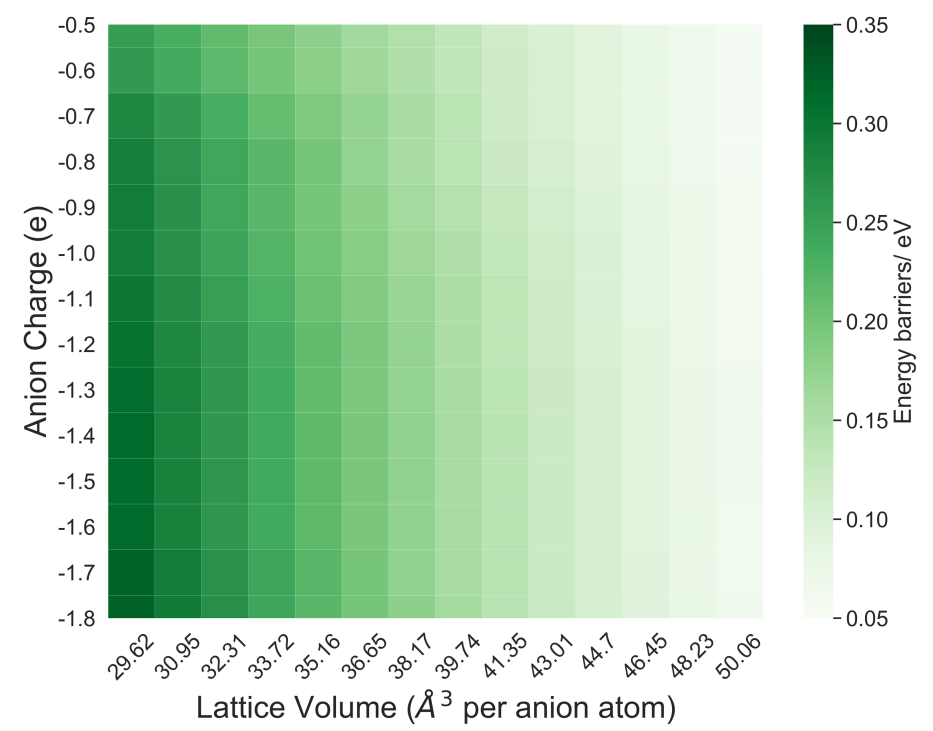

Figure 2. Heat maps of the calculated $\mathrm{E}_{\mathrm{a}}$ of lithium ion migration in the $b c c$-type sulfur anion sublattices along the Tet-Tet migration pathway with respect to anion charge and lattice volume. The corresponding data are also shown as the matrix table in Table S4.

\subsection{2 hcp-type anion sublattices}

Different from the less common $b c c$-type anion sublattices, there are both tetrahedral and octahedral interstitial sites in the $h c p$-type anion sublattice model (Figure 1b). Therefore, the discussions about the lithium ion migration in the $h c p$-type anion sublattice are organized based on three different pathways, including Tet-Tet, Oct-Oct and Oct-Tet.

\subsubsection{Tet-Tet pathway}


The calculated $\mathrm{E}_{\mathrm{a}}$ of lithium ion migration in the $h c p$-type sulfur(oxygen) anion sublattices along the direct Tet-Tet pathways with respect to anion charge and lattice volume are shown in Figure 3a, $\mathrm{S} 7, \mathrm{~S} 8$ and $\mathrm{S} 9$. It can be observed that $\mathrm{E}_{\mathrm{a}}$ varies from 0 to $0.5 \mathrm{eV}$ in the whole anion charge and lattice volume space for the Tet-Tet migration pathways in the hcp-type sulfur(oxygen) anion sublattice (Figure 3a and S7), whose ranges are similar to that of the Tet-Tet migration in the bcc-type sulfur(oxygen) anion sublattices (Figure 2 and S3) $(0-0.42 \mathrm{eV})$, and narrower than that of the $f c c$-type oxygen anion sublattice from 0.25 to $1.5 \mathrm{eV}$ (Figure S4a). Such a slightly smaller variation in the bcc-type anion sublattice may be due to the more distorted lithium-anion tetrahedrons (Figure S10) leading to a more frustrated energy landscape for lithium ion migration ${ }^{13}$. The maximum variation of $E_{a}$ with respect to different anion charges at any constant lattice volume is $\sim 0.09 \mathrm{eV}$ for the oxygen- and sulfur-anion sublattice (Figure S8 and S9), indicating that $\mathrm{E}_{\mathrm{a}}$ of lithium ion migration along the Tet-Tet pathways in lithium compounds with the hcp-type anion sublattices are much less sensitive to the anion charge, which is similar to the case of lithium compounds with the $b c c$-type anion sublattices.

However, different from the $b c c$-type sublattice, $\mathrm{E}_{\mathrm{a}}$ variations with respect to different anion charges at any constant lattice volume in the hcp-type anion sublattice are not monotonically increasing or decreasing (Figure S8 and S9), while $\mathrm{E}_{\mathrm{a}}$ variations are monotonic for the Tet-Tet migration pathways in the $b c c$-type anion sublattice (Figure S5 and S6). It may be because the different local environments of lithium tetrahedral sites and the diverse triangular anion bottlenecks (transition states) in the $b c c$ - and $h c p$-type anion sublattice, as shown in Figure S10. Taking the $h c p$-type sulfur anion sublattice as an example (Figure 3a and S9), the sulfur anion charges, ranging from $-0.8 e$ to $-1.3 e$, can significantly decrease $\mathrm{E}_{\mathrm{a}}$, while $\mathrm{E}_{\mathrm{a}}$ gradually increase when the more negative sulfur anion charges in the range of $[-1.3 e,-1.8 e]$. It's worth noting that discussing $\mathrm{E}_{\mathrm{a}}$ variation with respect to the much more positive anion charges in the range of $[-0.5 e,-0.8 e]$ make no sense due to the very low probability to get such small anion charges in lithium sulfides (Figure $\mathrm{S} 1)$. Moreover, it is found that the large lattice volumes can make low $\mathrm{E}_{\mathrm{a}}$ at any constant anion charge, and the effect of lattice volume on $\mathrm{E}_{\mathrm{a}}$ is larger than that of the anion charge for the Tet-Tet lithium ion migration. Thus, the moderate sulfur(oxygen) anion charges near $-1.3 e$ and especially large lattice volumes are beneficial to the fast lithium ion migration along the Tet-Tet migration pathways in lithium compounds with the hcp-type sulfur(oxygen) anion sublattices, although the effects of the Li-anion interaction depending on anion charge and lattice volume on the Tet-Tet lithium ion migration are limited. 
(a)

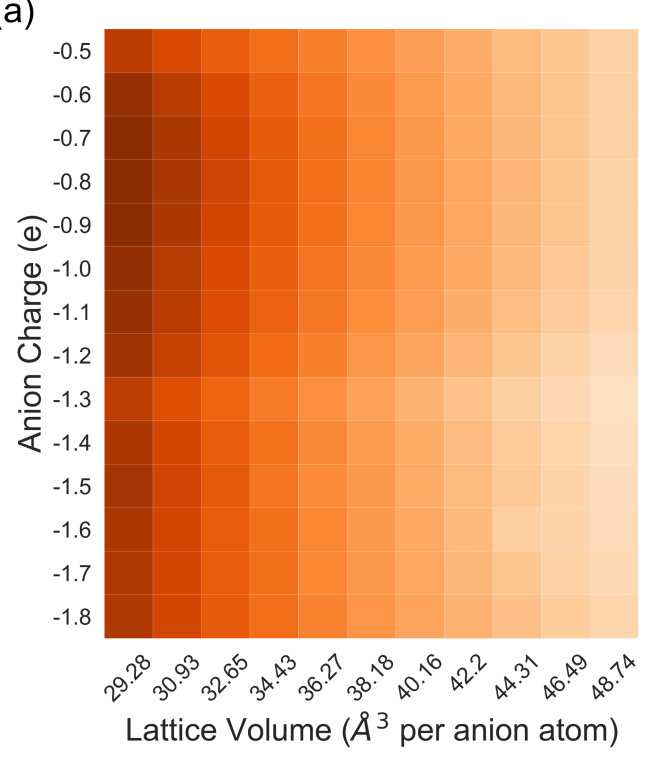

(c)

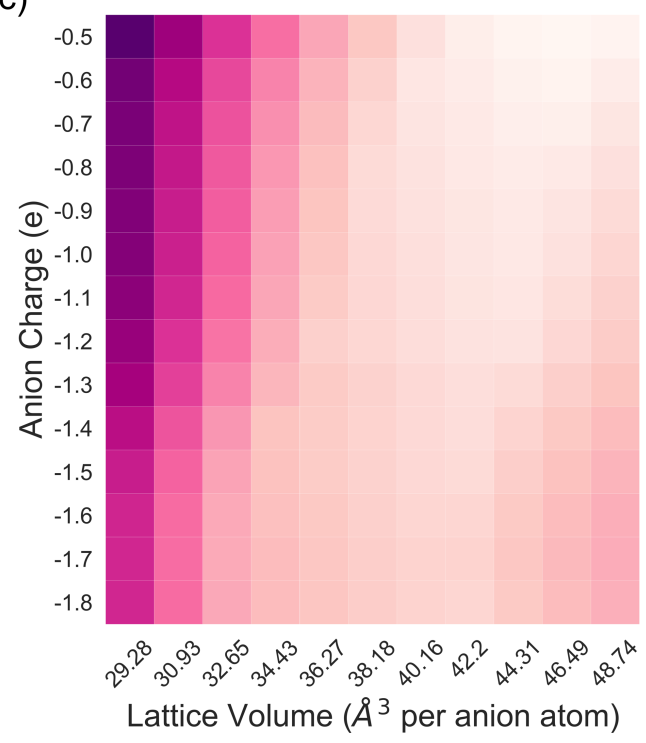

(b)
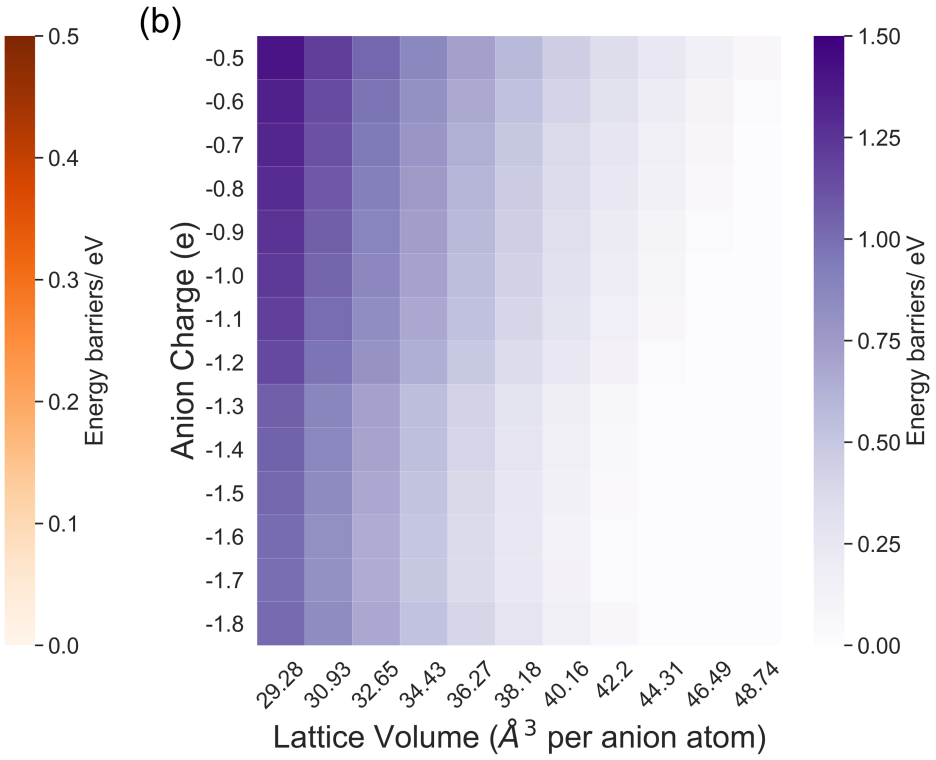

(d)
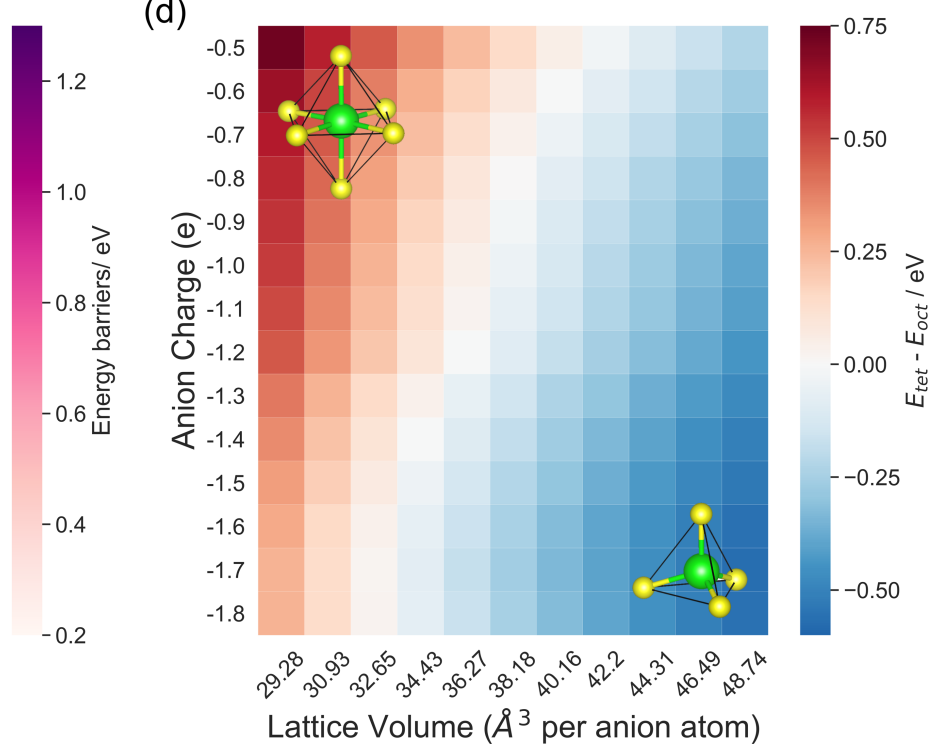

Figure 3. Heat maps of the calculated $\mathrm{E}_{\mathrm{a}}$ of lithium ion migration in the hcp-type sulfur anion sublattices along the (a) Tet-Tet, (b) Oct-Oct and (c) Oct-Tet (Tet-Oct) migration pathways with respect to anion charge and lattice volume, and (d) the energy differences of the occupied lithium between the Tet site and Oct site in the hcp-type sulfur anion sublattices with respect to anion charge and lattice volume. The corresponding data in heat maps are also shown as the matrix table in Table S5-S8.

\subsubsection{Oct-Oct pathway}

And then we focus on another direct lithium ion migration pathway in the hcp-type anion sublattices, namely Oct-Oct pathway. The calculated $\mathrm{E}_{\mathrm{a}}$ of lithium ion migration in the hcp-type 
sulfur(oxygen) anion sublattices along the Oct-Oct migration pathways with respect to anion charge and lattice volume are shown in Figure $3 b, \mathrm{~S} 11, \mathrm{~S} 12$ and $\mathrm{S} 13$. It can be found that $\mathrm{E}_{\mathrm{a}}$ variation ranging from 0 to $1.5 \mathrm{eV}$ in the whole anion charge and lattice volume space for the Oct-Oct migration pathways in the $h c p$-type sulfur(oxygen) anion sublattice is much wider than 0 to $0.5 \mathrm{eV}$ of the Tet-Tet migration pathways in the $b c c$ - and $h c p$-type sulfur(oxygen) anion sublattices. The maximum variation of $E_{a}$ with respect to different anion charges at any constant lattice volume is $\sim 0.4 \mathrm{eV}$ for the oxygen and sulfur anion sublattice (Figure S12 and S13), demonstrating that $\mathrm{E}_{\mathrm{a}}$ of lithium ion migration along the Oct-Oct pathways in lithium compounds with the hcp-type anion sublattices are sensitive to the variations of anion charge, and much different from the anion charge immunized Tet-Tet migration pathways. Interestingly, $\mathrm{E}_{\mathrm{a}}$ variations with respect to anion charge and lattice volume are monotonic. In the whole anion charge and lattice volume space, the more negative anion charges can reduce $\mathrm{E}_{\mathrm{a}}$ of lithium ion migration in the $h c p$-type anion sublattice along the Oct-Oct pathways at any constant lattice volume (Figure S12 and S13), and the large lattice volumes can make low $\mathrm{E}_{\mathrm{a}}$ at any constant anion charge. Over all, the Li-anion interactions depending on anion charge and lattice volume have great influences on $\mathrm{E}_{\mathrm{a}}$ of lithium ion migration along the Oct-Oct pathways in the hcp-type anion sublattices, and the more negative anion charges and large lattice volumes (lithium-anion bond lengths) are essential for achieving fast lithium ion migration along the Oct-Oct pathways in lithium compounds with the hcp-type anion sublattices.

\subsubsection{Oct-Tet pathway}

Lastly, we discuss the Oct-Tet pathway for lithium ion migration in the hcp-type anion sublattices, and it is the half of the Tet-Oct-Tet or Oct-Tet-Oct pathways, which are the most common lithium ion migration pathways in those lithium sulfides with the hcp-type sulfur anion sublattices ${ }^{40-42}$. The calculated $\mathrm{E}_{\mathrm{a}}$ of lithium ion migration along the Oct-Tet migration pathways, and the energy differences of lithium occupation between the Tet and Oct site ( $\mathrm{E}_{\text {tet-oct}}$ ) in the hcp-type sulfur(oxygen) anion sublattices with respect to anion charge and lattice volume are shown in Figure 3c, 3d, S14, S15 and S16. It can be observed that $E_{a}$ variation ranging from 0.2 to $1.4 \mathrm{eV}$ and Etet-oct variation from -0.76 to $0.9 \mathrm{eV}$ in the whole anion charge and lattice volume space for the Oct-Tet migration pathways in the hcp-type sulfur(oxygen) anion sublattices are very wide, indicating the anion charge and lattice volume have significant effects on $E_{a}$ and $E_{\text {tet-oct }}$ values for both the oxygen and sulfur anion systems. Within different lattice volume regions, the anion charges have different effects on $\mathrm{E}_{\mathrm{a}}$ for lithium ion migration along the Oct-Tet migration pathways 
in the $h c p$-type anion sublattices. For any specific lattice volume, the more negative anion charges consistently reduce $\mathrm{E}_{\text {tet-oct }}$ values (Figure $3 \mathrm{~d}$ and S14b), and stabilizing lithium-anion tetrahedra. At any constant anion charge, $E_{\text {tet-oct }}$ values vary from positive to negative, and the relative stabilities of lithium-anion tetrahedra gradually increase when lattice volumes get larger. The much negative anion charge and large lattice volume make lithium prefer to occupy the Tet sites in the hcp-type anion sublattices, and few negative anion charge and small lattice volume make lithium preferentially occupy the Oct sites (Figure $3 \mathrm{~d}$ and $\mathrm{S} 14 \mathrm{~b}$ ), as the same as the $f c c$-type oxygen anion sublattices (Figure S4b). For the Tet-Oct-Tet and Oct-Tet-Oct pathways, the corresponding transition state sites are Oct and Tet sites, respectively. Because of the relatively positive and negative energies of lithium ion occupying at Tet and Oct site $\left(\mathrm{E}_{\text {tet }}-\mathrm{E}_{\mathrm{oct}}>0,=0\right.$ and $\left.<0\right)$ with respect to different anion charges and lattice volumes, there are competitions of relative energy of the equilibrium site and saddle point for the Tet-Oct-Tet and Oct-Tet-Oct pathways in the hcp-and $f c c$-sublattices, consequently showing the opposite effect of anion charge on $\mathrm{E}_{\mathrm{a}}$ for Tet-Oct-Tet and Oct-Tet-Oct pathways, and the optimum lattice volumes for minimum $\mathrm{E}_{\mathrm{a}}$ at any one fixed anion charge (Figure 3c, S4a and S14a). While no anion charge and lattice volume optimizes minimum $\mathrm{E}_{\mathrm{a}}$ for the Tet-Tet and Oct-Oct pathways in the bcc-and hcp-type sublattices, due to no relative energy competitions between the equilibrium sites (Tet or Oct site) and high-energy saddle points, which are fully consistent with the previous work by Ceder et $\mathrm{al}^{11}$.

Facetgrid plots in Figure S15 and S16 show the anion charge dependent $\mathrm{E}_{\mathrm{a}}$ at different lattice volumes. Taking the sulfur anion sublattice as an example, when the sulfur anion lattices volume are small, e.g. with a value of $\sim 32.65 \AA$ /atom, the relative energies of $\mathrm{LiS}_{4}$ are all higher than those of $\mathrm{LiS}_{6}\left(\mathrm{E}_{\text {tet-oct }}>0\right.$, Figure $\left.3 \mathrm{~d}\right)$, indicating lithium is most stable at the Oct site and prefers to have the octahedral lithium occupation pattern with respect to small sulfur anion lattice volume, which is consistent with those $h c p$-type lithium sulfides with relative small lattice volumes ( $<39 \AA^{3} /$ atom) exhibiting octahedral lithium coordination environments (Table S1). Additionally, the more negative sulfur anion charges would lower the relative energies of $\mathrm{LiS}_{4}$, and hence reduce the corresponding $\mathrm{E}_{\mathrm{a}}$ for lithium ion migration along the Oct-Tet-Oct migration pathways in the $h c p$-type sulfur anion sublattices with smaller lattice volumes (Figure $3 \mathrm{~d}$ and S16). For the sulfur anion sublattice systems with medium lattice volumes, e.g. $\sim 36.27 \AA^{3} /$ atom, the relative energies of $\mathrm{LiS}_{4}$ are higher than those of $\mathrm{LiS}_{6}\left(\mathrm{E}_{\text {tet-oct }}>0\right.$, Figure $\left.3 \mathrm{~d}\right)$ for the systems with the less negative sulfur anion charges $\left(q_{\mathrm{S}} \in[-0.5 e,-1.1 e]\right)$. While the more negative sulfur anion charges $\left(q_{\mathrm{S}} \in\right.$ $[-1.1 e,-1.8 e])$ make the relative energies of $\mathrm{LiS}_{4}$ lower than those of $\mathrm{LiS}_{6}\left(\mathrm{E}_{\text {tet-oct }}<0\right)$, and thereby the Oct sites no longer keep stable. Thereby, with the negative sulfur anion charges increasing from 
-0.5 to $-1.8 e, \mathrm{E}_{\mathrm{a}}$ first decrease and then increase (Figure S16). At larger lattice volumes, e.g. 40.16 $\AA^{3} /$ atom, the relative energies of $\mathrm{LiS}_{4}$ are lower than those of $\mathrm{LiS}_{6}\left(\mathrm{E}_{\text {tet-oct }}<0\right.$, Figure $\left.3 \mathrm{~d}\right)$, indicating lithium prefers the Tet site at the large lattice volume, as shown in Table S1 that the hcp-type lithium sulfides with large sulfur lattice volumes ( $>39 \AA 3 /$ atom) showing the tetrahedral lithium occupation patterns. In short, the much negative anion charges would deliver high $\mathrm{E}_{\mathrm{a}}$ for the tetrahedral lithium ion migration along the Tet-Oct-Tet pathways in lithium compounds with hcp-type anion sublattices, but lower $\mathrm{E}_{\mathrm{a}}$ for the octahedral lithium ion migration along the Oct-Tet-Oct pathways.

For studying lithium ion migration in real lithium compounds with the $h c p$-type anion sublattices with respect to anion charge and lattice volume, we should first figure out the stable lithium occupation site and main lithium ion migration path. Table S1 and S2 clearly show that the lithium coordination environment in the most lithium sulfides with the $h c p$-type anion sublattices is tetrahedrally coordinated, and thereby the main lithium ion migration pathways in lithium sulfides are Tet-Tet and Tet-Oct-Tet. Because $\mathrm{E}_{\mathrm{a}}$ of lithium ion migration along the Tet-Tet pathways in the $h c p$-type anion sublattices is much less sensitive to the anion charge and lattice volume than that of the Tet-Oct-Tet pathway, so the overall lithium ion diffusion in compounds with hcp sulfur anion sublattices mainly exhibit the Tet-Oct-Tet pathway response to anion charge and lattice volume, and eventually the little negative anion charges would deliver low $E_{a}$ for the tetrahedral lithium ion migration in the $h c p$-type anion sublattices. Thus, the little negative anion charges and large lattice volumes (lithium-anion bond lengths) are necessary for achieving fast lithium ion migration in those lithium compounds with the hcp-type anion sublattices and stable tetrahedral lithium occupations.

The above analyses on the anion charge dependent $\mathrm{E}_{\mathrm{a}}$ for the hcp-type anion sublattice model are also validated by some real lithium sulfides. Figure 4 and Table S2 show some experimentally determined and theoretically calculated $E_{a}$ for the tetrahedral lithium ion migration in those lithium sulfides possessing hcp-type sulfur anion sublattices with respect to the sulfur anion Bader charges. As mentioned in section 3.1 (Figure S2), the Bader charges of lithium ion in different compounds don't change a lot, and thereby the anion charge variation of different lithium compounds can be regarded as resulting from the non-lithium cation element, which is fundamentally determined by the electronegativity difference between anion element and non-lithium cation element. In other words, the electronegativity of the non-lithium cation element $\mathrm{M}$ of Li-M-S compounds with $h c p$-type sulfur anion sublattices can significantly affect $\mathrm{E}_{\mathrm{a}}$ of lithium ion migration. It can be seen 
from Figure 4 that the non-lithium cation element $M$ has great impacts on $E_{a}$ for the tetrahedral lithium ion migration in those lithium sulfides with hcp-type sulfur anion sublattices. For example of the structurally similar $\mathrm{LiMS}_{2}$ (where $\mathrm{M}=\mathrm{In}$ and Ga) with the same space group (Pna2 ${ }_{1}$, No.33), the Ga element with relative larger electronegativity donates less electrons to its adjacent sulfur anions, and makes the smaller average Bader charge of sulfur anions in $\mathrm{LiGaS}_{2}$ than that of $\mathrm{LiInS}_{2}$, eventually leading to a lower $\mathrm{E}_{\mathrm{a}}$ of the tetrahedral lithium ion migration. Moreover, the same trends are also observed in $\mathrm{Li}_{3} \mathrm{MS}_{3}$ (where $\mathrm{M}=\mathrm{Al}$ and $\mathrm{Sb}$ ), $\mathrm{Li}_{3} \mathrm{MS}_{4}$ (where $\mathrm{M}=\mathrm{P}, \mathrm{As}$, and $\mathrm{Sb}$ ), and $\mathrm{Li}_{4} \mathrm{MS}_{4}$ (where $\mathrm{M}=\mathrm{Ge}$ and $\mathrm{Sn}$ ). For the most lithium sulfides with $h c p$-type sulfur anion sublattices in Table $\mathrm{S} 2$, there is no linear positive relationship between lattice volume and Li-S bond length (Figure $\mathrm{S} 17)$, which is quite different from the ideal anion sublattice model. For $\mathrm{Li}_{3} \mathrm{MS}_{4}(\mathrm{M}=\mathrm{P}, \mathrm{As}$, and $\mathrm{Sb})$ and $\mathrm{Li}_{4} \mathrm{MS}_{4}(\mathrm{M}=\mathrm{Ge}$ and $\mathrm{Sn})$, their Li-S bond lengths are found to be negatively related to the corresponding lattice volumes, and the larger $\mathrm{Li}-\mathrm{S}$ bond length of $\mathrm{Li}_{3} \mathrm{PS}_{4}$ further contribute to reduced $E_{a}$ together with the less negative charges of sulfur anion. But for $\operatorname{LiMS}_{2}(\mathrm{M}=\mathrm{In}$ and $\mathrm{Ga})$ and $\mathrm{Li}_{3} \mathrm{MS}_{3}(\mathrm{M}=\mathrm{Al}$ and $\mathrm{Sb})$, the larger Li-S bond lengths don't make the total $\mathrm{E}_{\mathrm{a}}$ reduced as expected, while the increased $E_{a}$ are mirrors of the more negative sulfur anion charges, indicating the weight of anion charge contributing to the total $E_{a}$ of those sulfides is very high. And yet there is no denying that the large lattice volume (lithium-anion bond length) can lower $\mathrm{E}_{\mathrm{a}}$ to a certain extent at any one fixed anion charge. The opposite effect of anion charge on $\mathrm{E}_{\mathrm{a}}$ for the Oct-Tet-Oct and Oct-Oct pathways in the hcp-type anion sublattices is confirmed by the experiment measured $\mathrm{E}_{\mathrm{a}}$ and ionic conductivities of those lithium chlorides with hcp-type chlorine anion sublattices (Table S3) ${ }^{21}$, 24. Above all, these $E_{a}$ variation tendencies with respect to the substituted non-lithium cation element $\mathrm{M}$ validate our anion charge-lattice volume maps of the hcp-type anion sublattice models. 

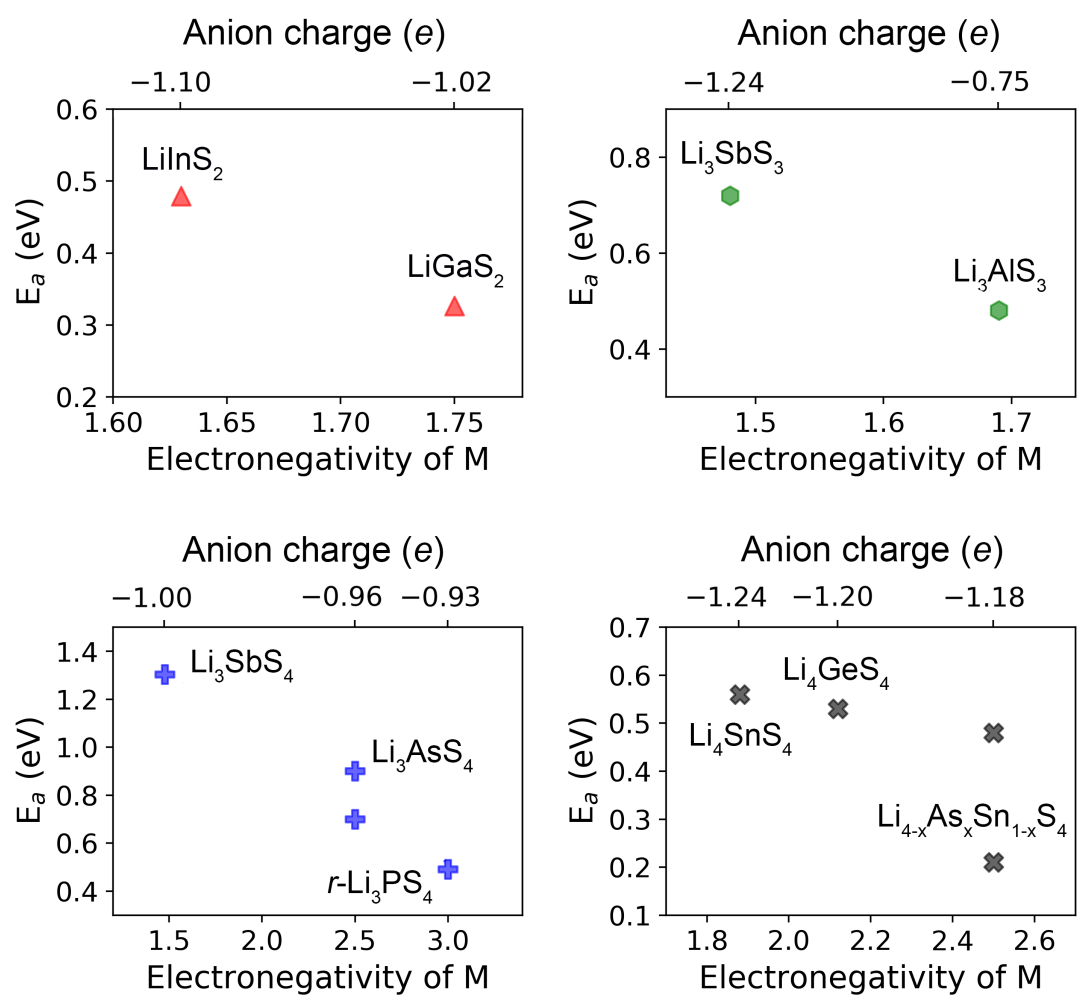

Figure 4. Experimentally determined and theoretically calculated activation energy barriers $E_{a}$ of the tetrahedral lithium ion migration in lithium sulfides possessing the hcp-type sulfur anion sublattices with respect to the electronegativity of non-lithium cation element $M$ and sulfur anion Bader charges ${ }^{40-42,45-48}$. Lithium sulfides in each subplot are structurally similar with the same space group. The corresponding data of $E_{a}$, anion charge and electronegativity of $M$ are also listed in Table S2.

\subsection{Design strategies for lithium superionic conductors}

In lithium compounds, the anion charges are significantly influenced by the non-lithium metal elements $^{26,38}$. The atomic radius and valence electron configuration of the non-lithium metal element determines its coordination environment and lattice volurme, eventually affecting the anion charge, lithium-anion bond length and lithium occupation pattern. Our model analyses of the $f c c-$, $b c c$ - and $h c p$-type anion sublattices clearly demonstrate that the anion charge and lattice volume (lithium-anion bond length) dramatically affect lithium ion migration especially for Oct-Tet and Oct-Oct pathways. Therefore, it makes sense to gain low $\mathrm{E}_{\mathrm{a}}$ for lithium ion migration by choosing the most suitable non-lithium metal element without changing the crystal structure sublattice a lot. 
Based on the deep understandings of the anion sublattice models (Figure 2, 3 and S3), two design strategies for developing new ternary $\mathrm{ABC}$ type lithium superionic conductors with $f c c$-, $b c c$ - and $h c p$-type anion sublattices are proposed here: (i) for the desired lithium superionic conductors with lithium (A) ion tetrahedral occupations, the small electronegativity difference between the anion elements $\mathrm{C}$ and the non-mobile cation elements $\mathrm{B}$ is necessary for obtaining fast lithium (A) ion migration along the Tet-Tet and Tet-Oct-Tet pathways (Figure 5a), and the proper non-mobile cation elements B should give preference to those main group elements located at the right top of the periodic table of elements with large electronegativities, which are close to but less than that of $\mathrm{C}$ elements, as shown in Figure $5 \mathrm{~b}$. The chemical components of the most sulfide-type superionic conductors with tetrahedral lithium ion migration along the Tet-Tet and Tet-Oct-Tet pathways, such as $\mathrm{Li}_{10} \mathrm{GeP}_{2} \mathrm{~S}_{12}{ }^{9}$, $\mathrm{Li}_{3} \mathrm{PS}_{4}{ }^{49-50}, \mathrm{Li}_{1+2 x} \mathrm{Zn}_{1-x} \mathrm{PS}_{4}{ }^{16}$ and $\mathrm{Li}_{2} \mathrm{CuPS}_{4}{ }^{51}$, are completely in conformity with this tetrahedron rule; (ii) for the desired lithium superionic conductors with lithium (A) ion octahedral occupations, the large electronegativity difference between the anion elements $\mathrm{C}$ and the non-mobile cation elements $\mathrm{B}$ is essential for getting fast lithium (A) ion migration along the Oct-Oct and Oct-Tet-Oct pathways (Figure 5a), and the promising non-mobile cation elements B should give preference to those subgroup transition-metal elements located at the left bottom of the periodic table with small electronegativities, as shown in Figure $5 b$. 
(a)

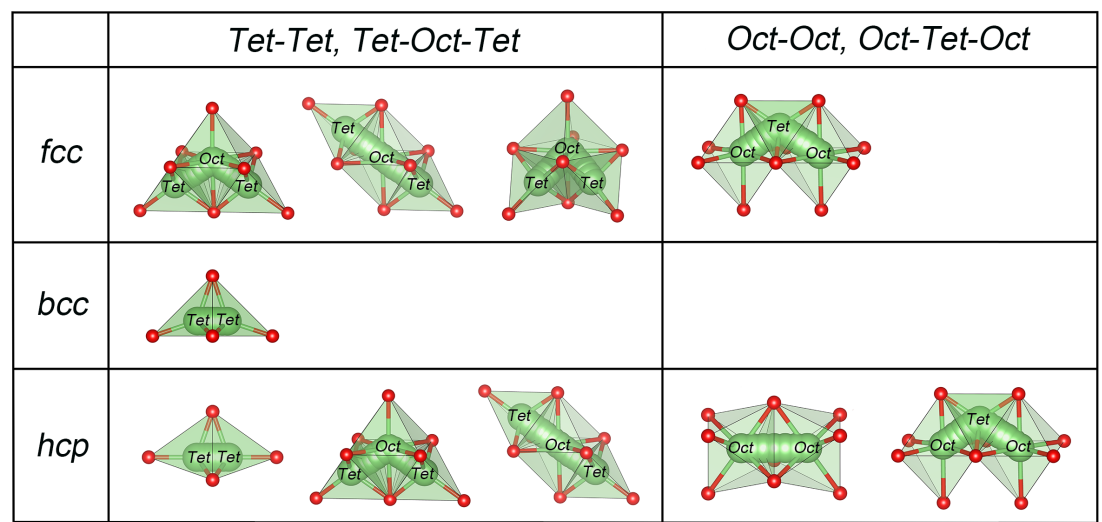

(b)

\begin{tabular}{|c|c|c|c|c|c|c|c|c|c|c|c|c|c|c|c|c|c|}
\hline H & & & & B & & super & ionic & $\mathrm{C}$ co & ndu & ictors & & & & & & & \\
\hline Li & $\mathrm{Be}$ & & & & & & & & & & & B & C & $\mathbf{N}$ & 0 & $F$ & \\
\hline $\mathrm{Na}$ & $\mathrm{Mg}$ & & & & & & & & & & & Al & $\mathrm{Si}$ & $\mathbf{P}$ & $\mathbf{s}$ & $\mathrm{Cl}$ & \\
\hline K & $\mathrm{Ca}$ & Sc & $\mathrm{Ti}$ & V & $\mathrm{Cr}$ & $\mathrm{Mn}$ & $\mathrm{Fe}$ & Co & $\mathrm{Ni}$ & $\mathrm{Cu}$ & $\mathrm{Zn}$ & Ga & Ge & As & $\mathrm{Se}$ & $\mathrm{Br}$ & $\mathrm{Kr}$ \\
\hline & $\mathrm{Sr}$ & $Y$ & $\mathrm{Zr}$ & $\mathrm{Nb}$ & Mo & Tc & $\mathbf{R u}$ & $\mathbf{R h}$ & $\mathbf{P d}$ & $\mathbf{A g}$ & Cd & In & Sn & Sb & $\mathrm{Te}$ & 1 & \\
\hline 1 & $\mathrm{Ba}$ & & Hf & $\mathrm{Ta}$ & $\mathbf{W}$ & $\operatorname{Re}$ & Os & Ir & $\mathrm{Pt}$ & $\mathrm{Au}$ & $\mathrm{Hg}$ & TI & $\mathrm{Pb}$ & $\mathbf{B i}$ & Po & At & $\mathrm{Rn}$ \\
\hline - & $\mathrm{Ra}$ & & Rf & $\mathrm{Db}$ & $\mathrm{Sg}$ & $\mathrm{Bh}$ & Hs & Mt & Ds & $\mathbf{R g}$ & Cn & $\mathrm{Nh}$ & FI & Mc & Lv & Ts & \\
\hline
\end{tabular}

La Ce Pr Nd Pm Sm Eu Gd Tb Dy Ho Er Tm Yb Lu

Ac Th Pa U Np Pu Am Cm Bk Cf Es Fm Md No Lr

A Mobile alkali-metal cation elements

B Main group non-mobile cation elements

B Subgroup transition-metal non-mobile cation elements

C Anion elements

Figure 5. (a) Migration pathways of the tetrahedrally and octahedrally occupied alkali-metal (A) ion in the $f c c$-, $b c c$ - and $h c p$-type anion sublattices. Considering the different relative positions of the initial and final Tet sites, there are three and two kinds of Tet-Oct-Tet pathways for the $f c c$ and $h c p$ type anion sublattices, respectively; (b) the recommended choices of the non-mobile cation element B (olive and orange areas) in the periodic table of element for achieving fast lithium (A) ion migration in the $\mathrm{ABC}$ ternary compounds. 


\section{Conclusions}

In this work, based on the density functional theory calculations and anion sublattice models, we have figured out the anion charge and lattice volume (lithium-anion bond length) dependent lithium ion diffusion in lithium compounds. The anion sublattice models clearly demonstrate that the anion charge and lattice volume dramatically affect $\mathrm{E}_{\mathrm{a}}$ for lithium ion migration, which is validated by some reported lithium sulfides. For the tetrahedrally occupied lithium, the less negative anion charge is, the lower the lithium ion migration barrier is. While for the octahedrally occupied lithium, the more negative anion charge is, the lower the lithium ion migration barrier is. The large lattice volume (lithium-anion bond length) can lower $\mathrm{E}_{\mathrm{a}}$ to a certain extent. The activation energy depends more strongly on the lattice volume than on the anion charge. Lithium ion direct migrations along the direct Tet-Tet pathway in the $b c c$ - or hcp-type anion sublattice are less sensitive to anion charge and lattice volume than other pathways. The much negative anion charge and large lattice volume make lithium prefer to occupy the Tet sites in the $f c c$ - and $h c p$-type anion sublattices, and few negative anion charge and small lattice volume make lithium preferentially occupy the Oct sites. There are opposite effects of anion charge on $E_{a}$ and optimum lattice volumes for minimum $E_{a}$ of lithium ion migration along the Tet-Oct-Tet and Oct-Tet-Oct pathways in the hcp-type sublattices.

Moreover, general design strategies for developing advanced lithium superionic conductors were proposed based on the full understandings of anion sublattice model. Choosing the most suitable non-mobile cation element without changing the crystal structure sublattice to get the desired electronegativity difference between the anion element and non-mobile cation element, can eventually achieve low $\mathrm{E}_{\mathrm{a}}$ for lithium ion migration. For the desired lithium superionic conductors with lithium ion tetrahedral occupations, the small electronegativity difference between the anion element and four-coordinated non-mobile cation element is necessary for obtaining fast lithium ion migration along the Tet-Tet and Tet-Oct-Tet pathways, and the fine non-mobile cation element should give preference to those elements located at the right top of the periodic table of elements with large electronegativity. For the lithium superionic conductors with lithium ion octahedral occupations, the large electronegativity difference between the anion element and six-coordinated non-mobile cation element is essential for getting fast lithium ion migration along the Oct-Oct and Oct-Tet-Oct pathways, and the fine non-mobile cation element should give preference to the elements located at the left bottom of the periodic table with small electronegativity. 


\section{Acknowledgements}

This work is supported by the National Natural Science Foundation of China (51602196), Shanghai Automotive Industry Corporation (1714), and Materials Genome Initiative Center at Shanghai Jiao Tong University. Z. $\mathrm{Xu}$ is much grateful to the support of the China Scholarship Council (CSC, scholarship No. 201906230117). All simulations were performed at the Shanghai Jiao Tong University High Performance Computing Center.

\section{Supporting information}

Scatter distributions of anion Bader charge and lattice volume of lithium compounds; Histogram distributions of Bader charges of lithium ion in compounds; Heat maps and facetgrid plots of calculated $\mathrm{E}_{\mathrm{a}}$ for oxygen and sulfur anion sublattices; Matched $b c c$ and $h c p$ anion sublattices for lithium sulfides from the MP database; Experimentally determined and theoretically calculated $\mathrm{E}_{\mathrm{a}}$ of tetrahedral Li ion migration in lithium sulfides with $h c p$-type sulfur anion sublattices; Scatter distributions of lattice volumes and average Li-S bond lengths of lithium sulfides with tetrahedral lithium occupations and $h c p$-type sulfur anion sublattices.

\section{Author's information}

Corresponding Authors

*E-mail: hong.zhu@sjtu.edu.cn

Notes

The authors declare no competing financial interest.

\section{References}

1. Pomerantseva, E.; Bonaccorso, F.; Feng, X.; Cui, Y.; Gogotsi, Y., Energy storage: The future enabled by nanomaterials. Science 2019, 366, 969.

2. Dunn, B.; Kamath, H.; Tarascon, J. M., Electrical energy storage for the grid: a battery of choices. Science 2011, 334, 928-935. 
3. Harper, G.; Sommerville, R.; Kendrick, E.; Driscoll, L.; Slater, P.; Stolkin, R.; Walton, A.; Christensen, P.; Heidrich, O.; Lambert, S.; Abbott, A.; Ryder, K.; Gaines, L.; Anderson, P., Recycling lithium-ion batteries from electric vehicles. Nature 2019, 575 (7781), 75-86.

4. Zhang, Z.; Shao, Y.; Lotsch, B.; Hu, Y.-S.; Li, H.; Janek, J.; Nazar, L. F.; Nan, C.-W.; Maier, J.; Armand, M.; Chen, L., New horizons for inorganic solid state ion conductors. Energy \& Environmental Science 2018, 8, 1945-1976.

5. Xabier Jude, G. G. E., Chunmei Li, Lide M. Rodriguez-Martinez, Heng Zhang, a. M. A., Opportunities for Rechargeable Solid-State Batteries Based on Li-Intercalation Cathodes. Joule 2018, 2, 1-17.

6. Sun, C.; Liu, J.; Gong, Y.; Wilkinson, D. P.; Zhang, J., Recent advances in all-solid-state rechargeable lithium batteries. Nano Energy 2017, 33, 363-386.

7. Murugan, R.; Thangadurai, V.; Weppner, W., Fast Lithium Ion Conduction in Garnet-Type $\mathrm{Li}_{7} \mathrm{La}_{3} \mathrm{Zr}_{2} \mathrm{O}_{12}$. Angew. Chem. Int. Ed. 2007, 46 (41), 7778-7781.

8. Takada, K.; Tansho, M.; Yanase, I.; Inada, T.; Kajiyama, A.; Kouguchi, M.; Kondo, S.; Watanabe, M., Lithium ion conduction in $\mathrm{LiTi}_{2}\left(\mathrm{PO}_{4}\right)_{3}$. Solid State Ionics 2001, 139 (3), 241-247.

9. Kamaya, N.; Homma, K.; Yamakawa, Y.; Hirayama, M.; Kanno, R.; Yonemura, M.; Kamiyama, T.; Kato, Y.; Hama, S.; Kawamoto, K.; Mitsui, A., A lithium superionic conductor. Nature materials 2011, 10 (9), 682-686.

10. Seino, Y.; Ota, T.; Takada, K.; Hayashi, A.; Tatsumisago, M., A sulphide lithium super ion conductor is superior to liquid ion conductors for use in rechargeable batteries. Energy Environ. Sci. 2014, 7 (2), 627-631.

11. Wang, Y.; Richards, W. D.; Ong, S. P.; Miara, L. J.; Kim, J. C.; Mo, Y.; Ceder, G., Design principles for solid-state lithium superionic conductors. Nature materials 2015, 14, 1026.

12. He, X.; Bai, Q.; Liu, Y.; Nolan, A. M.; Ling, C.; Mo, Y., Crystal Structural Framework of Lithium Super-Ionic Conductors. Advanced Energy Materials 2019, 9 (43), 1902078.

13. Di Stefano, D.; Miglio, A.; Robeyns, K.; Filinchuk, Y.; Lechartier, M.; Senyshyn, A.; Ishida, H.; Spannenberger, S.; Prutsch, D.; Lunghammer, S.; Rettenwander, D.; Wilkening, M.; Roling, B.; Kato, Y.; Hautier, G., Superionic Diffusion through Frustrated Energy Landscape. Chem 2019, 5, $1-11$.

14. Blatov, V. A.; Shevchenko, A. P.; Proserpio, D. M., Applied Topological Analysis of Crystal Structures with the Program Package ToposPro. Crystal Growth \& Design 2014, 14 (7), 3576-3586. 15. Eremin, R. A.; Kabanova, N. A.; Morkhova, Y. A.; Golov, A. A.; Blatov, V. A., High-throughput search for potential potassium ion conductors: A combination of 
geometrical-topological and density functional theory approaches. Solid State Ionics 2018, 326, 188-199.

16. Richards, W. D.; Wang, Y.; Miara, L. J.; Kim, J. C.; Ceder, G., Design of $\mathrm{Li}_{1+2 x} \mathrm{Zn}_{1-\mathrm{x}} \mathrm{PS}_{4}$, a new lithium ion conductor. Energy \& Environmental Science 2016, 9 (10), 3272-3278.

17. Kato, Y.; Hori, S.; Saito, T.; Suzuki, K.; Hirayama, M.; Mitsui, A.; Yonemura, M.; Iba, H.; Kanno, R., High-power all-solid-state batteries using sulfide superionic conductors. Nature Energy 2016, 1 (4), 16030.

18. Fisher, C. A. J.; Hart Prieto, V. M.; Islam, M. S., Lithium Battery Materials LiMPO $4(M=M n$, $\mathrm{Fe}, \mathrm{Co}$, and Ni): Insights into Defect Association, Transport Mechanisms, and Doping Behavior. Chem. Mater. 2008, 20 (18), 5907-5915.

19. Gardiner, G. R.; Islam, M. S., Anti-Site Defects and Ion Migration in the $\mathrm{LiFe}_{0.5} \mathrm{Mn}_{0.5} \mathrm{PO}_{4}$ Mixed-Metal Cathode Material. Chem. Mater. 2010, 22 (3), 1242-1248.

20. Wang, S.; Bai, Q.; Nolan, A. M.; Liu, Y.; Gong, S.; Sun, Q.; Mo, Y., Lithium Chlorides and Bromides as Promising Solid-State Chemistries for Fast Ion Conductors with Good Electrochemical Stability. Angew. Chem. Int. Ed. 2019, 58 (24), 8039-8043.

21. Asano, T.; Sakai, A.; Ouchi, S.; Sakaida, M.; Miyazaki, A.; Hasegawa, S., Solid Halide Electrolytes with High Lithium-Ion Conductivity for Application in $4 \mathrm{~V}$ Class Bulk-Type All-Solid-State Batteries. Adv. Mater. 2018, 30, 1803075.

22. Krauskopf, T.; Muy, S.; Culver, S. P.; Ohno, S.; Delaire, O.; Shao-Horn, Y.; Zeier, W. G., Comparing the Descriptors for Investigating the Influence of Lattice Dynamics on Ionic Transport Using the Superionic Conductor $\mathrm{Na}_{3} \mathrm{PS}_{4-\mathrm{x}} \mathrm{Se}_{\mathrm{x}} . J$. Am. Chem. Soc. 2018, 140 (43), 14464-14473.

23. Kraft, M. A.; Ohno, S.; Zinkevich, T.; Koerver, R.; Culver, S. P.; Fuchs, T.; Senyshyn, A.; Indris, S.; Morgan, B. J.; Zeier, W. G., Inducing High Ionic Conductivity in the Lithium Superionic Argyrodites $\mathrm{Li}_{6+} \mathrm{P}_{1-\mathrm{x}} \mathrm{Ge}_{\mathrm{x}} \mathrm{S}_{5} \mathrm{I}$ for All-Solid-State Batteries. J. Am. Chem. Soc. 2018, 140 (47), 16330-16339.

24. Muy, S.; Voss, J.; Schlem, R.; Koerver, R.; Sedlmaier, S. J.; Maglia, F.; Lamp, P.; Zeier, W. G.; Shao-Horn, Y., High-Throughput Screening of Solid-State Li-Ion Conductors Using Lattice-Dynamics Descriptors. iScience 2019, 16, 270-282.

25. Kraft, M. A.; Culver, S. P.; Calderon, M.; Bocher, F.; Krauskopf, T.; Senyshyn, A.; Dietrich, C.; Zevalkink, A.; Janek, J.; Zeier, W. G., Influence of Lattice Polarizability on the Ionic Conductivity in the Lithium Superionic Argyrodites $\mathrm{Li}_{6} \mathrm{PS}_{5} \mathrm{X}(\mathrm{X}=\mathrm{Cl}, \mathrm{Br}, \mathrm{I})$. J. Am. Chem. Soc. 2017, 139 (31), 10909-10918. 
26. Xu, Z. M.; Bo, S. H.; Zhu, H., $\mathrm{LiCrS}_{2}$ and $\mathrm{LiMnS}_{2}$ Cathodes with Extraordinary Mixed Electron-Ion Conductivities and Favorable Interfacial Compatibilities with Sulfide Electrolyte. ACS applied materials \& interfaces 2018, 10, 36941-36953.

27. Xu, Z.; Chen, X.; Liu, K.; Chen, R.; Zeng, X.; Zhu, H., Influence of Anion Charge on Li Ion Diffusion in a New Solid-State Electrolyte, $\mathrm{Li}_{3} \mathrm{LaI}_{6}$. Chem. Mater. 2019, 31 (18), 7425-7433.

28. Blöchl, P. E., Projector augmented-wave method. Physical Review B 1994, 50 (24), 17953-17979.

29. Verma, P.; Truhlar, D. G., Status and Challenges of Density Functional Theory. Trends in Chemistry 2020, 2 (4), 302-318.

30. Kohn, W.; Sham, L. J., Self-Consistent Equations Including Exchange and Correlation Effects. Phys. Rev. 1965, 140 (4A), A1133-A1138.

31. Perdew, J. P.; Burke, K.; Ernzerhof, M., Generalized Gradient Approximation Made Simple. Phys. Rev. Lett. 1996, 77 (18), 3865-3868.

32. Monkhorst, H. J.; Pack, J. D., Special points for Brillouin-zone integrations. Physical Review B 1976, 13 (12), 5188-5192.

33. Henkelman, G.; Jónsson, H., Improved tangent estimate in the nudged elastic band method for finding minimum energy paths and saddle points. The Journal of Chemical Physics 2000, 113 (22), 9978-9985.

34. Curtarolo, S.; Setyawan, W.; Hart, G. L. W.; Jahnatek, M.; Chepulskii, R. V.; Taylor, R. H.; Wang, S.; Xue, J.; Yang, K.; Levy, O.; Mehl, M. J.; Stokes, H. T.; Demchenko, D. O.; Morgan, D., AFLOW: An automatic framework for high-throughput materials discovery. Computational Materials Science 2012, 58, 218-226.

35. Nishitani, Y.; Adams, S.; Ichikawa, K.; Tsujita, T., Evaluation of magnesium ion migration in inorganic oxides by the bond valence site energy method. Solid State Ionics 2018, 315, 111-115.

36. Chen, H.; Wong, L. L.; Adams, S., SoftBV-a software tool for screening the materials genome of inorganic fast ion conductors. Acta Crystallographica Section B Structural Science, Crystal Engineering and Materials 2019, 75 (1), 18-33.

37. Li, K.; Xue, D., Estimation of electronegativity values of elements in different valence states. $J$ Phys Chem A 2006, 110 (39), 11332-7.

38. Krauskopf, T.; Culver, S. P.; Zeier, W. G., Bottleneck of Diffusion and Inductive Effects in Li10Ge1-xSnxP2S12. Chem. Mater. 2018, 30 (5), 1791-1798.

39. Culver, S. P.; Koerver, R.; Krauskopf, T.; Zeier, W. G., Designing Ionic Conductors: The Interplay between Structural Phenomena and Interfaces in Thiophosphate-Based Solid-State Batteries. Chem. Mater. 2018, 30 (13), 4179-4192. 
40. Murayama, M.; Sonoyama, N.; Yamada, A.; Kanno, R., Material design of new lithium ionic conductor, thio-LISICON, in the $\mathrm{Li}_{2} \mathrm{~S}-\mathrm{P}_{2} \mathrm{~S}_{5}$ system. Solid State Ionics 2004, 170 (3-4), 173-180.

41. Sahu, G.; Lin, Z.; Li, J.; Liu, Z.; Dudney, N.; Liang, C., Air-stable, high-conduction solid electrolytes of arsenic-substituted $\mathrm{Li}_{4} \mathrm{SnS}_{4}$. Energy Environ. Sci. 2014, 7 (3), 1053-1058.

42. Kanno, R., Synthesis of a new lithium ionic conductor, thio-LISICON-lithium germanium sulfide system. Solid State Ionics 2000, 130 (1-2), 97-104.

43. Rong, Z.; Malik, R.; Canepa, P.; Sai Gautam, G.; Liu, M.; Jain, A.; Persson, K.; Ceder, G., Materials Design Rules for Multivalent Ion Mobility in Intercalation Structures. Chem. Mater. 2015, 27 (17), 6016-6021.

44. He, X.; Zhu, Y.; Mo, Y., Origin of fast ion diffusion in super-ionic conductors. Nature communications 2017, 8, 15893.

45. Rao, F.-Y.; Ning, F.-H.; Jiang, L.-W.; Zeng, X.-M.; Wu, M.-S.; Xu, B.; Ouyang, C.-Y., First principle study of $\mathrm{LiXS}_{2}(\mathrm{X}=\mathrm{Ga}$, In) as cathode materials for Li ion batteries. Chinese Physics $B$ 2016, 25 (2), 028202.

46. Gamon, J.; Duff, B. B.; Dyer, M. S.; Collins, C.; Daniels, L. M.; Surta, T. W.; Sharp, P. M.; Gaultois, M. W.; Blanc, F.; Claridge, J. B.; Rosseinsky, M. J., Computationally Guided Discovery of the Sulfide $\mathrm{Li}_{3} \mathrm{AlS}_{3}$ in the Li-Al-S Phase Field: Structure and Lithium Conductivity. Chem. Mater. 2019, 31 (23), 9699-9714.

47. Al-Qawasmeh, A.; Holzwarth, N. A. W., Computational Study of Li Ion Electrolytes Composed of $\mathrm{Li}_{3} \mathrm{AsS}_{4}$ Alloyed with $\mathrm{Li}_{4} \mathrm{GeS}_{4}$. J. Electrochem. Soc. 2016, 163 (9), A2079-A2088.

48. Kwak, H.; Park, K. H.; Han, D.; Nam, K.-W.; Kim, H.; Jung, Y. S., Li ${ }^{+}$conduction in air-stable Sb-Substituted $\mathrm{Li}_{4} \mathrm{SnS}_{4}$ for all-solid-state Li-Ion batteries. J. Power Sources 2020, 446.

49. Tachez, M.; Malugani, J.-P.; Mercier, R.; Robert, G., Ionic conductivity of and phase transition in lithium thiophosphate $\mathrm{Li}_{3} \mathrm{PS}_{4}$. Solid State Ionics 1984, 14 (3), 181-185.

50. Homma, K.; Yonemura, M.; Kobayashi, T.; Nagao, M.; Hirayama, M.; Kanno, R., Crystal structure and phase transitions of the lithium ionic conductor $\mathrm{Li}_{3} \mathrm{PS}_{4}$. Solid State Ionics 2011, 182 (1), 53-58.

51. Xu, Z.; Chen, R.; Zhu, H., A $\mathrm{Li}_{2} \mathrm{CuPS}_{4}$ superionic conductor: a new sulfide-based solid-state electrolyte. Journal of Materials Chemistry A 2019, 7 (20), 12645-12653. 